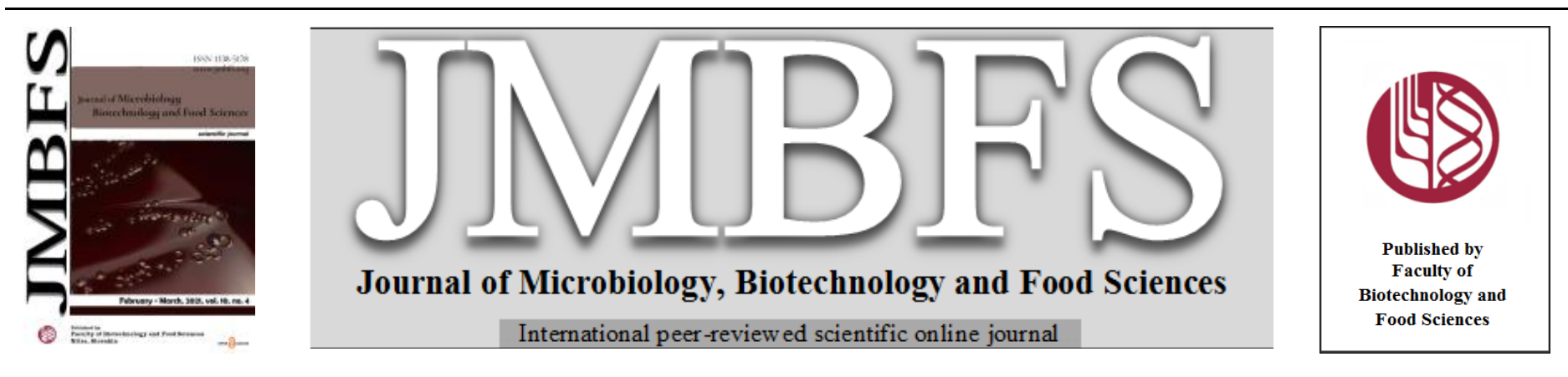

\title{
OPTIMIZATION OF FACS SORTING FOR THE IMPROVEMENT OF LIVESTOCK SEMEN QUALITY
}

\author{
Jaromír Vašíček ${ }^{* 1,2}$, Andrea Svoradová ${ }^{1}$, Andrej Baláži ${ }^{1}$, Rastislav Jurčik ${ }^{1}$, Marián Macháč², Alexander Ostró ${ }^{3}$, Peter Chrenek ${ }^{1,2}$
}

Address(es): Ing. Jaromír Vašǐček, PhD.

${ }^{1}$ NPPC, Research Institute for Animal Production in Nitra, Institute of Farm Animal Genetics and Reproduction, Hlohovecká 2, 95141 Lužianky, Slovak Republic, phone number: +421 376546600

${ }^{2}$ Slovak University of Agriculture in Nitra, Faculty of Biotechnology and Food Science, Department of Biochemistry and Biotechnology, Tr. A. Hlinku 2, 94976 Nitra, Slovak Republic.

${ }^{3}$ Pavol Jozef Šafárik University in Kosice, Faculty of medicine, Department of Gynaecology and Obstetrics, Tr. SNP 1, 04011 Košice, Slovak Republic.

*Corresponding author: jaromir.vasicek@nppc.sk

doi: 10.15414/jmbfs.2021.10.4.697-705

ARTICLE INFO

Received 30. 9. 2020

Revised 23. 11. 2020

Accepted 2. 12. 2020

Published 1. 2. 2021

Regular article

open $\partial_{\text {AcCESS }}$

\begin{abstract}
Fluorescence-activated cell sorting (FACS) of spermatozoa was mainly used for sex sorting. Recently, FACS has been used to eliminate DNA-damaged human spermatozoa using YO-PRO-1 staining. Fragmentation of sperm DNA is considered as one of the reasons of male infertility. Moreover, YO-PRO-1 can effectively mark apoptotic as well as dead spermatozoa. Till now, only two FACS instruments were used for the spermatozoa sorting. However, both of them are not more commercially available from different reasons. In this study, we used novel FACSMelody Cell Sorter for the elimination of apoptotic and dead cells from the rabbit and ram semen samples in order to improve their overall quality. Briefly, semen samples were stained using YO-PRO-1 dye (apoptotic and dead cells) and/or propidium iodide (PI; only dead cells). Three different sorting experiments were performed: E1 - YO-PRO-1 and PI stained rabbit sperm cells were sorted into the tubes containing $1 \mathrm{ml}$ of PBS; E2 - PI stained rabbit sperm cells were sorted into tubes that were washed with FBS prior adding PBS; and E3 - YO-PRO-1 and PI stained ram sperm cells were sorted into tubes washed with FBS prior adding PBS. As a sheath fluid sterile PBS was used. All samples, control (before sorting), negatively and positively sorted fractions, were analysed using CASA for motility assessment. Moreover, all sorted samples were re-stained with PI for viability assessment. In conclusion, elimination of dead $\left(\mathrm{PI}^{+}\right)$sperm from rabbit samples might improve their quality, since their progressive motility increased significantly $(\mathrm{P}<0.001)$ after sorting from 40 to $65 \%$. However, ram spermatozoa seem to be sensitive to sorting procedure thus further optimisation of this procedure is required.
\end{abstract}

Keywords: FACS, sperm quality, motility, viability, YO-PRO-1, PI

\section{INTRODUCTION}

Fluorescence-activated cell sorting (FACS) of sperm cells is used mainly for sperm sexing since turn off the 80s and 90s (Johnson et al., 1989; Johnson et al., 1994; Johnson et al., 1999; Johnson, 2000; Garner, 2006) using Beltsville sorting technology. This method is based on the differences in DNA content of $\mathrm{X}$ - and Y-bearing sperm cells that are stained with Hoeschst 33342 dye (Seidel Jr, 2007; Sharpe and Evans, 2009). Beginning with the first report in rabbits (Johnson et al., 1989) to date, this method has become a commercially used in the bovine (Frijters et al., 2009), swine (Garcia et al., 2007; Vazquez et al., 2009) and ovine (de Graaf et al., 2006; Leahy et al., 2010) industry. In recent years, the Magnetic-activated cell sorting (MACS) has been used to improve the quality of human or livestock semen based on the elimination of apoptotic spermatozoa via binding of Annexin V-conjugated nanoparticles to externalized phosphatidylserine (Grunewald et al., 2001). This method can enhance the reproductive outcomes in human clinical practice (Said et al., 2006; OsegueraLópez et al., 2019), but not in livestock production as reported in rabbits (Vasicek et al., 2014) or boar (Mrkun et al., 2014). On the other hand, it was observed that bull sperm cells with damaged membrane can be effectively removed using propidium iodide-conjugated magnetic nanoparticles (Fox $\boldsymbol{e t}$ al., 2012). However, the reproductive outcomes regarding this method are unknown. As an alternative to MACS, FACS sorting of YO-PRO-1 positive cells were recently used to remove apoptotic cells and thus reduced the number of DNAfragmented spermatozoa in order to improve the outcomes in assisted reproduction (Ribeiro et al., 2013; De Geyter et al., 2019). Thus, beside the sperm sexing FACS sorting might be used also to improve semen quality. However, for both sorting procedures only two instruments were used as mentioned in published studies (de Graaf et al., 2007; da Silva et al., 2013; Ribeiro et al., 2013; Quan et al., 2015; de Geyter et al., 2019). First of them, a MoFLo SX DP sorter (Beckman Coulter, Miami, FI, USA) with a patented nozzle specially improved for sperm sorting is not available for research use. The second one, a BD Influx flow cytometer (BD Biosciences, San Jose, CA, USA) intended for research applications is not more available at the market. For this reason, a novel flow-cytometric sorter FACSMelody (BD, New Jersey, USA) with constant pressure and nozzle size was tested in the present study.

The main objective of this preliminary study was to optimize the sorting of rabbit and ram spermatozoa using FACSMelody sorter in order to enhance the output semen quality by removal of apoptotic and dead cells while maintaining and/or improving their motility parameters.

\section{MATERIAL AND METHODS}

\section{Animals}

Clinically healthy and sexually mature rabbits $(n=6)$ and rams $(n=6)$ were used in this study as follow: six bucks of New Zealand White rabbit breed, one ram of Native Wallachian sheep breed, two rams of Improved Wallachian sheep breed and two rams of Slovak Dairy sheep breed. All animals were reared at the breeding facility (NPPC, RIAP Nitra, Lužianky, Slovak Republic) and semen samples were collected as described previously (Kuželová $\boldsymbol{e t ~ a l . , ~ 2 0 1 7 ; ~ B a l a ́ z ̌ i ~} \boldsymbol{e}$ al., 2020). The experiments were carried out in accordance with the Code of Ethics of the EU Directive 2010/63/EU for animal experiments (https://ec.europa.eu/environment/chemicals/lab_animals/legislation_en.htm)

\section{Experimental design}

Rabbit and ram semen samples were sorted using YO-PRO-1 dye staining apoptotic cells and dead cells and/or propidium iodide (PI) that stains only dead cells. Three different sorting experiments were performed. At first, both YOPRO-1 and PI stained rabbit sperm cells were sorted into the $5 \mathrm{ml}$ round-bottom 
BD Falcon polystyrene tubes (BD, New Jersey, USA) containing $1 \mathrm{ml}$ of phosphate-buffered saline (PBS without $\mathrm{Ca}$ and $\mathrm{Mg}$; Biosera, France) (Experiment $1-$ E1). Since tube walls could be sticky for sorted cells, PI stained rabbit sperm cells were sorted into Falcon tubes that were washed with fetal bovine serum (FBS; Sigma-Aldrich, Germany) prior the addition of PBS (Experiment 2 - E2). At last, both YO-PRO-1 and PI stained ram sperm cells were sorted into Falcon tubes washed with FBS as in E2 (Experiment 3 - E3).

\section{Computer assisted sperm analysis (CASA)}

Fresh semen samples (control samples) were diluted and analysed using CASA system (Sperm Vision ${ }^{\mathrm{TM}}$, MiniTübe, Germany). For each sample, concentration of spermatozoa $\left(10^{9}\right.$ per $\mathrm{mL}$ ), percentage of total motility (motility $>5 \mu \mathrm{m} / \mathrm{s}$ ) and progressive motility (motility $>20 \mu \mathrm{m} / \mathrm{s}$ ) of spermatozoa were measured as described previously (Kuželová et al., 2017; Baláži et al., 2020). The motility parameters were observed also in stained (YO-PRO-1 and PI) samples before sorting and subsequently in negative fractions of sorted samples in order to assess the effect of staining and sorting on semen quality.

\section{YO-PRO-1 and PI staining}

Semen samples were diluted in PBS to a final concentration of $10^{7} \mathrm{sperm} / \mathrm{mL}$ and incubated with YO-PRO-1 (Molecular Probes, Eugene, Oregon, USA) at final concentration of $100 \mu \mathrm{mol} / \mathrm{L}$ for $15 \mathrm{~min}$. in the dark at room temperature. Samples were washed after incubation in PBS at $600 \mathrm{x} \mathrm{g}$ for $5 \mathrm{~min}$. and resuspended in $1 \mathrm{ml}$ of PBS for sorting procedure. Other diluted semen samples were mixed with staining solution of PI (Molecular Probes, Eugene, Oregon, USA) at final concentration of $50 \mu \mathrm{g} / \mathrm{mL}$ and sorted immediately without subsequent incubation and washing steps.

\section{Fluorescent activated cell sorting (FACS)}

The stained samples were sorted using BD FACSMelody Cell Sorter (BD, New Jersey, USA) equipped with 488-nm blue direct diode laser $(20 \mathrm{~mW}), 640$-nm red direct diode laser $(40 \mathrm{~mW})$ and $100 \mu \mathrm{m}$ nozzle operating at $23 \mathrm{psi}$. The lasers were aligned using calibration beads provided by $\mathrm{BD}$ prior to each sorting. Sterile PBS $\left(137 \mathrm{mM} \mathrm{NaCl}, 2.7 \mathrm{mM} \mathrm{KCl}, 1.5 \mathrm{mM} \mathrm{KH}_{2} \mathrm{PO}_{4}\right.$ and $8.1 \mathrm{mM} \mathrm{Na}_{2} \mathrm{HPO}_{4}$. $12 \mathrm{H}_{2} \mathrm{O} ; \mathrm{pH}=7.4$ ) was used as sheath fluid for sorting and additional analyses. To eliminate debris and noise, the sperm cells were gated using a forward and side scatter and only singlets (single cells) were chosen in order to exclude the agglutinated sperm from the analysis (Figure 1A and 2A). The average sorting rate was 500-3500 events/s. Sorting was continued until 200,000 to 500,000 sperm cells negative for YO-PRO-1 or PI were collected not exceeding the total sorting volume of $0.5-1 \mathrm{ml}$. Aliquot of negative fraction from each sort was analysed using CASA as mentioned before. Both sorted fractions (positive and negative) of each sort were stained again with PI at final concentration of 50 $\mu \mathrm{g} / \mathrm{mL}$ and analysed again $(10,000$ cells) in order to control the viability and/or efficiency of sorting (Figure 1B and 2B). All sorting experiments were acquired and evaluate using BD FACSChorus Software (BD, New Jersey, USA).
A
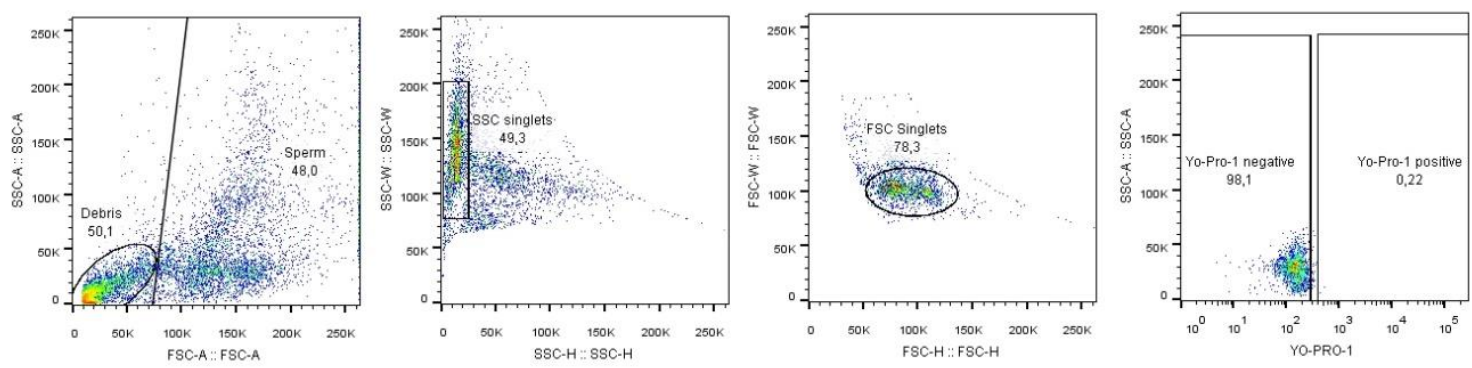

B
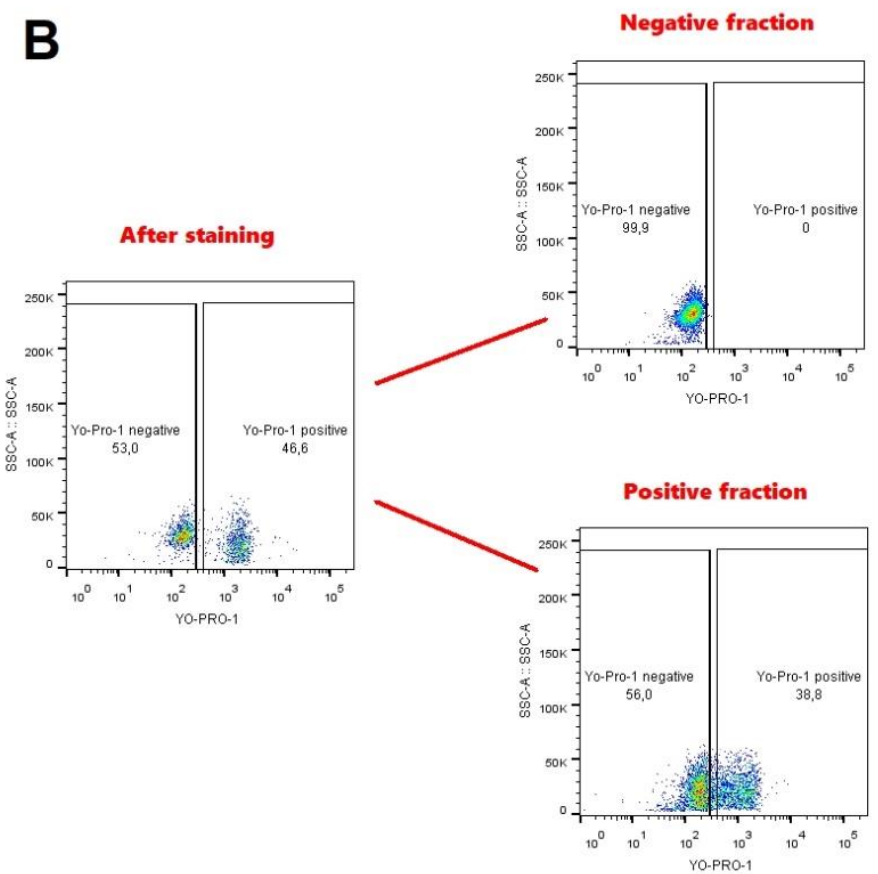

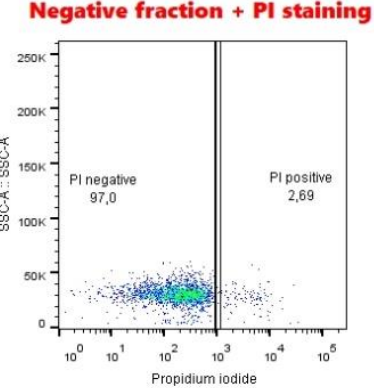

Positive fraction + PI staining

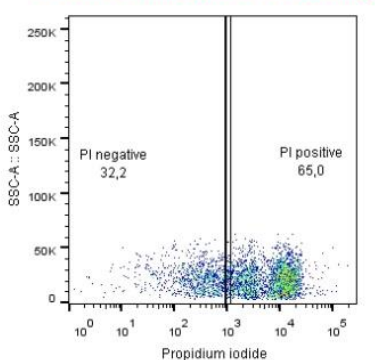

Figure 1 Sorting strategy of YO-PRO-1 stained rabbit spermatozoa: A) Sperm cells were gated in order to eliminate debris and only singlets were chosen for analysis and sorting. B) YO-PRO-1 stained cells were sorted according to the positivity into negative and positive fraction, analysed for YO-PRO-1 positivity and after-stain with PI for viability assessment. 
A
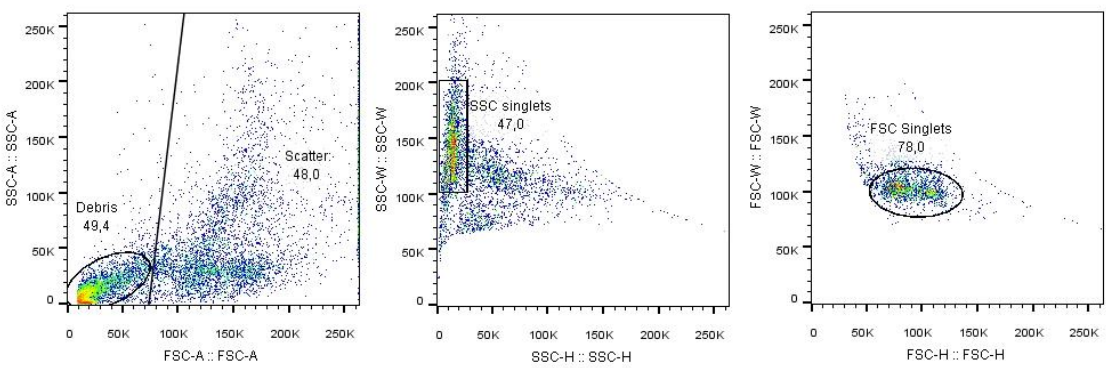

B
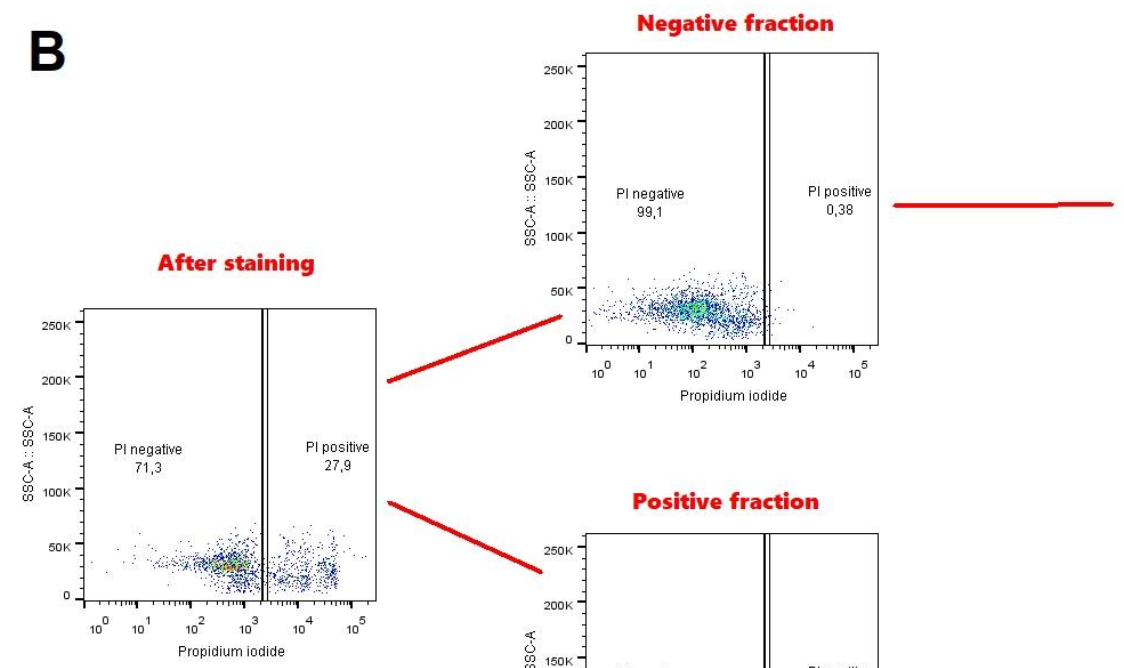
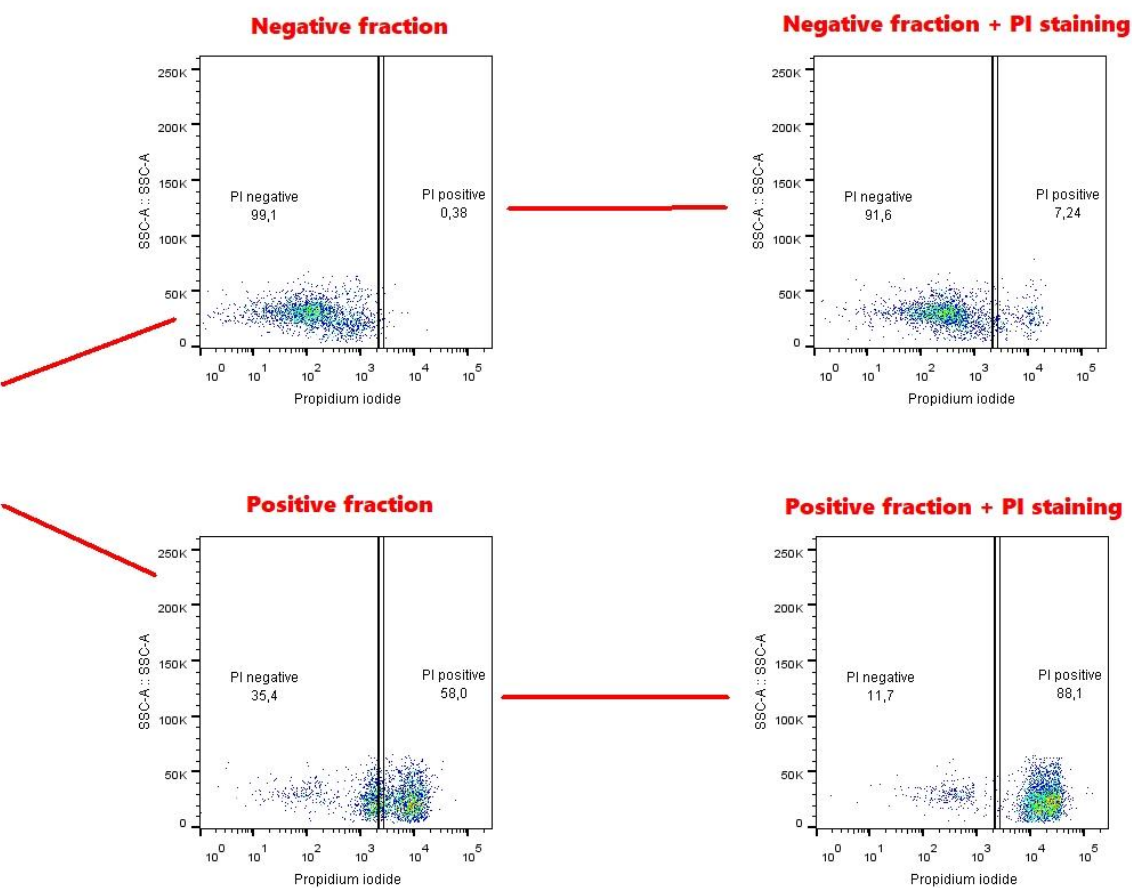

Figure 2 Sorting strategy of PI stained rabbit spermatozoa: A) Sperm cells were gated in order to eliminate debris and only singlets were chosen for analysis and sorting. B) PI stained cells were sorted according to the positivity into negative and positive fraction, analysed for PI positivity to check the sorting efficiency and afterstain again with PI for viability assessment.

\section{Statistical analysis}

Obtained results, sorting efficiency and viability of sorted sperm cells were as well as motility parameters (CASA), were evaluated using the SigmaPlot software (Systat Software Inc., Germany) with one-way ANOVA (Holm-Sidak method) and expressed as the means \pm SEM. $P$-values at $P<0.05$ were considered as statistically significant.

\section{RESULTS}

\section{Experiment 1}

In this experiment, rabbit sperm cells were sorted into the prepared tubes with PBS after staining with YO-PRO-1 or PI in order to remove apoptotic or dead cells, respectively (Fig. 3). A high sorting efficiency was achieved for both of
YO-PRO-1 and PI as negative fractions showed zero positivity (Fig. 3A, C) However, the percentage of YO-PRO- $1^{+}$cells in positive fraction did not change when compared to the control sample (Fig. 3A). On the other hand, $\mathrm{PI}^{+}$cells increased from $11 \%$ to about $50 \%$ in positive fraction in comparison to control (Fig. 3C). Concerning the viability of sorted samples, the proportion of dead cells $\left(\mathrm{PI}^{+}\right)$reached about $90 \%$ in negative and $70 \%$ in positive fractions of YO-PRO1 sorted spermatozoa (Fig. 3B). The viability of negative fraction after PI sorting was significantly better in comparison to the positive fraction, although the percentage of dead cells achieved almost 45\% (Fig. 3D). The motility analyses of sorted spermatozoa corresponded with the decrease in cell viability (Fig. 3E). In both sorting experiments, a slight decrease in total and progressive movement of spermatozoa was observed immediately after staining. Moreover, all reported motility parameters dropped to zero values in negative fractions after both sorting procedures. Thus, the sorting technique presented in E1 could not be useful to improve the quality of spermatozoa in terms of their viability and motility. 

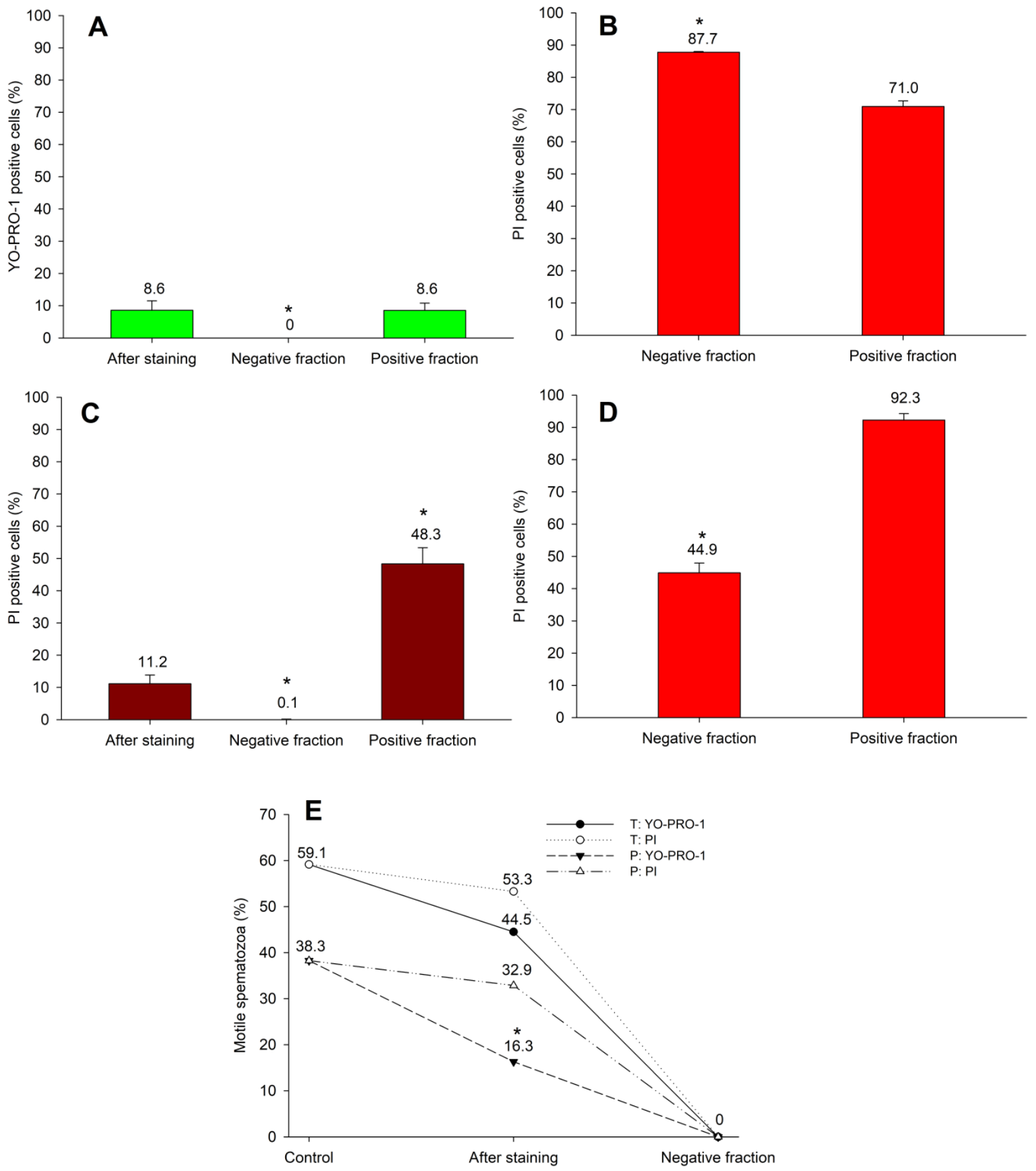

Figure 3 Sorting of rabbit spermatozoa (Experiment 1)

A) Sorting efficiency of YO-PRO-1 sorted spermatozoa (*different at P $<0.05$ ); B) viability of YO-PRO-1 sorted spermatozoa (*different at P<0.001); C) Sorting efficiency of PI sorted spermatozoa (*negative fraction different to stained sample at $\mathrm{P}<0.05$ and positive fraction different to stained sample at $\mathrm{P}<0.001$ ); $\mathrm{D}$ ) viability of PI sorted spermatozoa (*different at P<0.001); E) Sperm motility before and after staining and sorting. $\mathrm{T}$ - total and $\mathrm{P}$ - progressive motility of YO-PRO-1 and PI stained and sorted spermatozoa (*different to control sample at $\mathrm{P}<0.05)$.

\section{Experiment 2}

In E2 experiment, tubes used for retaining the sorted spermatozoa were washed with FBS prior to the sorting of PI stained rabbit spermatozoa (Fig. 4) in order to increase the viability and yield of sorted sperm cells. Again, the sorting efficiency was high as negative fraction showed zero PI positivity. However, PI positivity in positive fraction increased only twofold from 9 to $17 \%$ (Fig. 4A). On the other hand, the cell viability retained significantly high (about 90\%) in negative fraction compared to the positive fraction (Fig. 4B). This tendency was confirmed by motility analysis, since both total and progressive $(\mathrm{P}<0.05)$ motility of sorted spermatozoa in negative fraction increased in comparison to the control sample (Fig. 4C). 

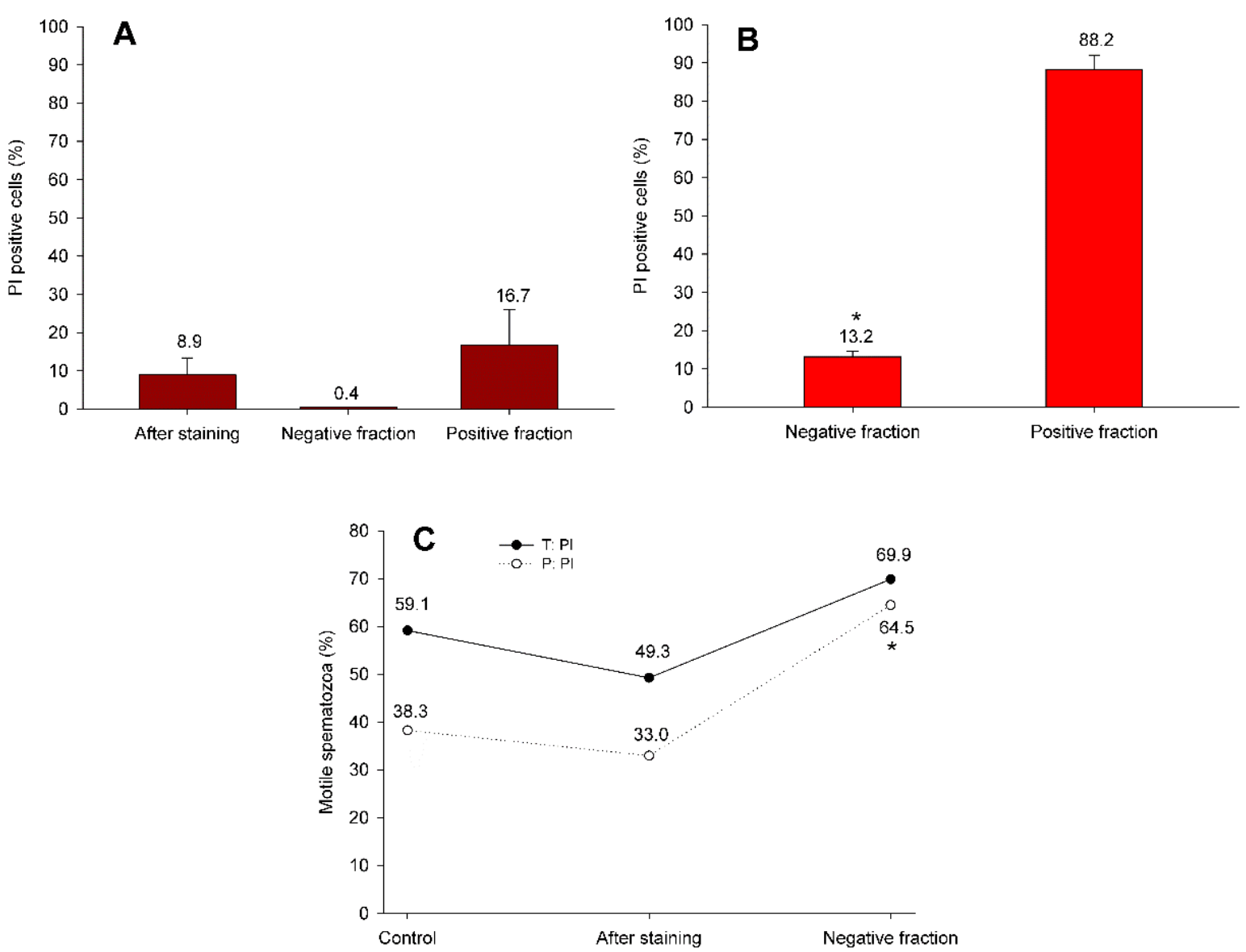

Figure 4 Sorting of rabbit spermatozoa (Experiment 2)

A) Sorting efficiency of PI sorted spermatozoa; B) viability of PI sorted spermatozoa (*different at $\mathrm{P}<0.001)$; C) Sperm motility before and after staining and sorting. $\mathrm{T}$ - total and $\mathrm{P}$ - progressive motility of PI stained and sorted spermatozoa (*different to control sample at $\mathrm{P}<0.05$ ).

\section{Experiment 3}

In the last experiment, ram spermatozoa were sorted into FBS washed tubes (Fig 5 ) as in E2. In this experiment both YO-PRO-1 and PI stained ram spermatozoa were sorted in order to confirm the sorting procedure that seems to be optimized for rabbit spermatozoa as shown in E2. Although, repeatedly high sorting efficiencies were noticed in negative fractions of YO-PRO-1 and PI sorted sperm (Fig. 5A, C), significantly decreased positivity was observed in YO-PRO-1 positive fraction or no change in the proportion of positive cells was noticed in $\mathrm{PI}$ positive fraction when compared to the control samples. The viability of both YO-PRO-1 and PI sorted ram sperm cells was significantly better in negative fractions in comparison to the positive fractions (Fig. 5B, D), but did not significantly improve in comparison to the control sample $(35 \%$ and $63 \%$, respectively in comparison to the control sample viability of $49 \%$; Fig. 5C). On the other hand, although the motility parameters decreased after staining, the motility values of both YO-PRO-1 and PI sorted spermatozoa were restored to the basal values before sorting without any improvement (Fig. 5E). 

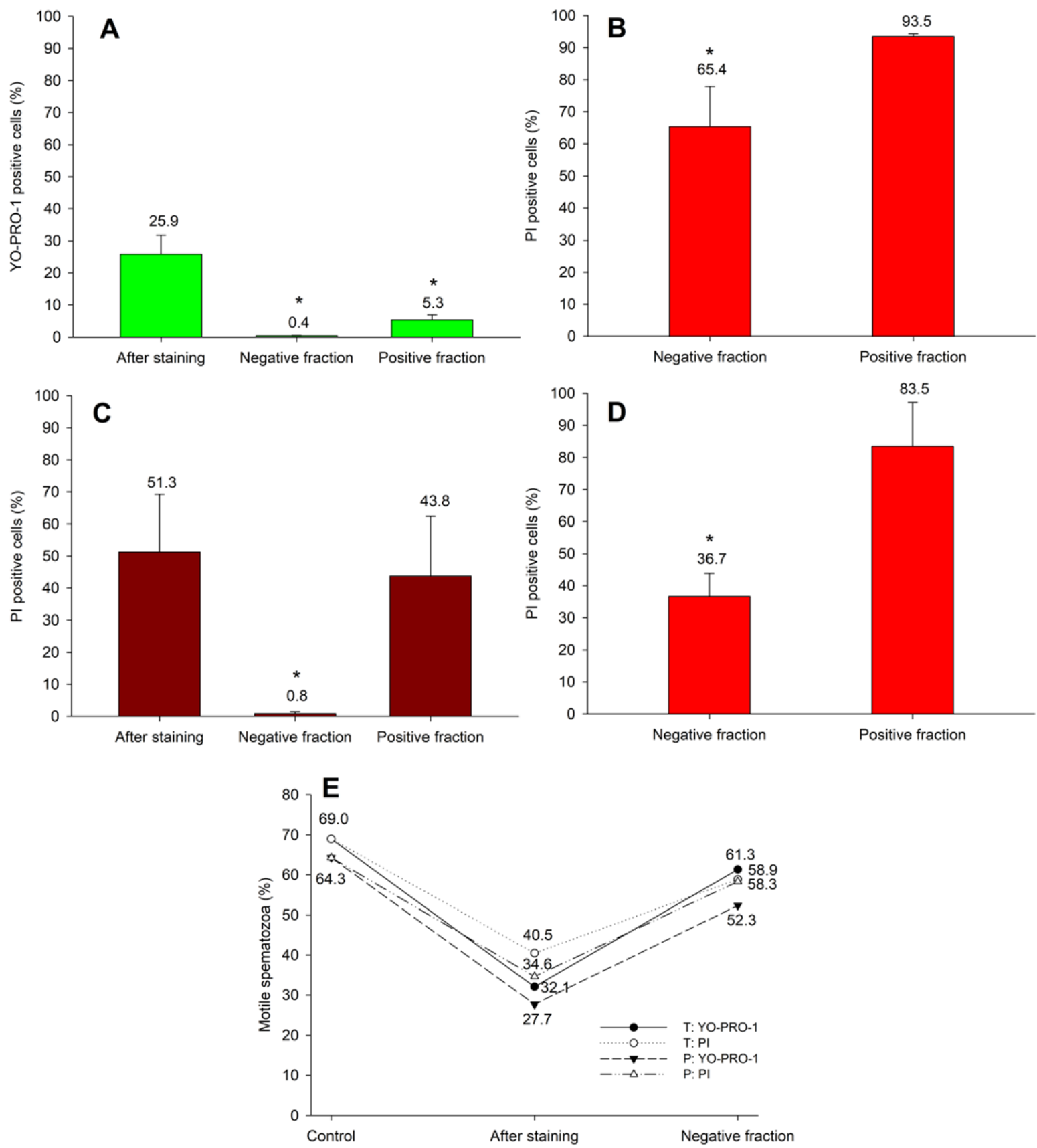

Figure 5 Sorting of ram spermatozoa (Experiment 3 )

A) Sorting efficiency of YO-PRO-1 sorted spermatozoa (*different to stained sample at $\mathrm{P}<0.05$ ); B) viability of YO-PRO-1 sorted spermatozoa (*different at $\mathrm{P}<0.05$ ); C) Sorting efficiency of PI sorted spermatozoa (*different at P<0.05); D) viability of PI sorted spermatozoa (*different at $\mathrm{P}<0.05)$; E) Sperm motility before and after staining and sorting. $\mathrm{T}-$ total and $\mathrm{P}$ - progressive motility of YO-PRO-1 and PI stained and sorted spermatozoa.

\section{DISCUSSION}

For the first time, FACSMelody sorter was used to sort apoptotic and dead spermatozoa of different animal species for the purpose of potential improvement of the semen quality. Until recently, FACS sorting procedure of spermatozoa was mainly used for sex sorting. However, a few recent studies (Ribeiro et al., 2013; De Geyter et al., 2019) showed that using a YO-PRO-1 staining and FACS sorting the proportion of DNA-fragmented spermatozoa in human semen samples can be reduced to $5 \%$ as a result of apoptotic cell removal. In general, fragmentation of nuclear DNA is one of the main features of apoptosis (Ribeiro et al., 2013). Increased amount of apoptotic sperm cells within the semen that is associated with higher number of spermatozoa with DNA fragmentation can resulted in male infertility (Aziz et al., 2007; Schulte et al., 2010; Aitken et al., 2011; Varshini $\boldsymbol{e t}$ al., 2012; Zorn $\boldsymbol{e t}$ al., 2012) as well as in early pregnancy loss (Zini et al., 2008; Robinson et al., 2012). Thus, DNA integrity is crucial not only for successful fertilization, but also for further development of embryos (Morris et al., 2002; Virro et al., 2004; Enciso et al., 2012). YO-PRO-1 staining has been shown to be an effective, simple, fast and trustworthy method for the quantification of spermatozoa apoptosis (Martin et al., 2004; Martin et al.,
2007) and seems to be nontoxic when used for short-term incubation (less than 3 days) (Idziorek et al., 1995; Gawlitta et al., 2004). In addition, this dye costains apoptotic as well as dead spermatozoa (Ribeiro et al., 2013).

In the presented study, we tried to optimize the sorting of YO-PRO-1 (apoptotic and dead cells) and PI (only dead cells) stained rabbit and ram spermatozoa. We used nontoxic YO-PRO-1 dye that requires incubation and washing steps Oppositely, the PI staining omits incubation, as this toxic agent can be harmful to cells and long-term culture could stain also live cells (Zhao et al., 2010). In all experiments (E1-3), positively stained sperm cells were effectively sorted as negative fractions did not exceed $1 \%$ of positive cell. High purity of YO-PRO-1 sorted spermatozoa (about 98.3\%) was similarly observed by Ribeiro et al. (2013). On the other hand, the positive fractions either YO-PRO-1 or PI sorted cells (E1-E3) achieved only 5-50\% purity (understand as \% of positive cells). However, reanalysis with PI staining of positively sorted fractions in all E1-E3 revealed noticeably higher (70-94\%) percentage of dead cells when compared to those that were just sorted (5-50\%). Thus, it seems that dyes YO-PRO-1 (data not shown) and/or PI were somehow washed out from the positively stained cells during the sorting procedure. This phenomenon might be possibly caused by the pressure of sorting instrument that impacts the sorted cells. Unfortunately, the 
published studies did not observe the purity of positive fractions after sorting and so we could not confirm this hypothesis by other findings.

In general, highly diluted spermatozoa are during sorting procedure exposed to the potential dye toxicity, electrical charge, different media and/or mechanical forces (Leahy et al., 2011). Additional damaging factors for the sorted spermatozoa can be their acceleration through the microfluidic system, laser beam exposure, their distribution into the droplets and at last their falling down to collecting tubes (Gosalvez et al., 2011). Previous studies aimed on sex sorted spermatozoa revealed that high pressures (about $50 \mathrm{psi}$ ) have detrimental effect on their viability and motility (Suh et al., 2005), as well as on embryo development (Campos-Chillon and de la Torre, 2003) and fertility (Seidel $\boldsymbol{e}$ al., 2003). However, those effects were eliminated by reducing the sorting pressures below 30 psi (Suh et al., 2005). The reanalysis of sorted positive and negative fractions in all our experiments with PI enabled to assess the viability of sorted spermatozoa and thus to evaluate the possible harmful impact of sorting procedure. First of all, it has to be mentioned that the original sheath fluid designed for FACS sorters contains some type of preservatives that may have adverse cytotoxic effect on certain type of cells. To eliminate this effect, a sterile preservative-free PBS was used as sheath fluid as it can be found in severa published studies focused on sperm sorting. Secondly, we observed significant drop in viability of negatively sorted rabbit spermatozoa in E1 when compared to sample before sorting (from $90 \%$ to $13 \%$ for YO-PRO-1 or 55\% for PI). On the contrary, the viability of PI sorted rabbit sperm (E2) in negative fraction was comparable to sample prior sorting (about 90\%). Since, we used the same staining procedure in both experiments (E1 and E2), washing the collection tubes with FBS prior the sorting most probably facilitates the dropping of spermatozoa into the tubes and thus retained their initial viability. Similarly, the viability of sorted ram spermatozoa in E3 varied in negative fractions between 40-60\% (YOPRO-1 and PI, respectively) in comparison to control sample with $50 \%$ of viability. However, it means that either FBS washing of the collecting tubes did not improve significantly the sperm viability after sorting.

In this study, FACSMelody sorter with constant pressure at 23 psi was used. According to the above-mentioned studies, this pressure size might not negatively influence the viability or motility of sorted spermatozoa. Similarly, Ribeiro et al. (2013) reported only about 6-7\% of damaged spermatozoa in human YO-PRO-1 negatively sorted spermatozoa after re-staining with YOPRO-1 when a sorting pressure at 20 psi was used. Moreover, they observed about $25 \%$ of dead sperm in negatively sorted fraction; however, they did not state the viability of spermatozoa before sorting. In addition, they noticed that motility of those spermatozoa decreased after sorting. Another study of da Silva et al. (2013) aimed on sex sorting revealed that this procedure increased the membrane permeability of stallion spermatozoa. The authors demonstrated that the viability of sex sorted spermatozoa was reduced from 70 to $60 \%$. At the same time, a significant decrease in the spermatozoa motility was observed after sorting, but even more also already after the staining of samples before the sorting itself. Other authors (Quan et al., 2015) observed that independently of the dye concentration (Hoechst 33342 in this study) or the staining duration, the staining procedure itself negatively affects the sperm motility and viability, as well as the presence of apoptotic spermatozoa in the stained ram semen sample In all our experiments (E1-E3), we also found a decrease in sperm motility immediately after staining of rabbit or ram semen samples. Since the YO-PRO-1 dye is relatively nontoxic for short-term use as it was demonstrated above, probably the washing steps and the dilution of spermatozoa itself might negatively affect their motility. In case of PI, a slight toxic effect of this dye could be taken into consideration as a reason for the decline in the sperm motility. However, more important fact is that the progressive motility of rabbit sperm cells increased significantly after the FACS elimination of $\mathrm{PI}^{+}$cells (E2). Moreover, the sorted ram spermatozoa got back the motility parameters that were similar to their initial motility prior sorting and staining (E3). On the other hand, the majority of sorted spermatozoa in E1 were non-motile, that correlates with their pure viability after sorting probably caused by omitting the FBS washing step of collecting tubes. In general, we did not observe major improvement (except for E2) either reduction (except for E1) in the sperm motility after sorting procedure. However, some immobilizing effect of FACS procedure that is correlated with the initial sperm quality was previously observed by Ribeiro $e t$ al. (2013). Much earlier, Auger et al. (1993) suggested according to their findings that only sperm with disturbed function could be immobilized or even killed by FACS.

In summary, from the presented experiments (E2 and E3) we can conclude that rabbit sperm quality might be improved by the elimination of dead spermatozoa via FACS. On the other hand, although the viability of PI sorted ram spermatozoa slightly increased, the viability of ram spermatozoa that were sorted using $\mathrm{YO}$ PRO-1 decreased and their motility in both cases was not improved. Thus, ram spermatozoa seems to be much more sensitive to the sorting procedure as rabbit sperm cells. In general, it has been already proved that ram spermatozoa are very sensitive to any oxidative damage (Hamilton $\boldsymbol{e t}$ al., 2016) or cold shock (Mendoza et al., 2013). Anyway, it seems that PI sorting of ram spermatozoa seems to be less sensitive to sperm cells, maybe due to the omitting of washing steps. Moreover, De Geyter et al. (2019) stated that removal YO-PRO- $1^{+}$human spermatozoa with damaged DNA using FACS did not significantly enhance the fertility results of assisted reproduction.

Broadly speaking, YO-PRO-1 could be a convenient alternative for removal of apoptotic cells in contrary to Annexin V staining, since this dye labels both apoptotic cells with intact membrane as well as cells with comprised membrane (Idziorek et al., 1995; Ribeiro et al., 2013) thus omitting the staining of dead cells with additional necrotic marker such as propidium iodide. On the other hand, although the PI staining technique is easy and relatively quick, the potential carcinogenic effect of PI on the stained living cells should be taken into consideration. However, at the present sorting of apoptotic cells using YO-PRO-1 staining is possible only via FACS as there is no available similar commercial kit conjugated with nanoparticles as for example the Annexin V MicroBead kit (Miltenyi Biotec, Germany) for the MACS removal of apoptotic cells. Although, the latter mentioned kit is successfully used in human assisted reproduction (Said et al., 2006); the largescale application of this method for the improvement of livestock semen quality has been proven as unsatisfying for rabbit (Vasicek $\boldsymbol{e} t$ al., 2014) or boar (Mrkun et al., 2014). On the other hand, the MACS technique offers a much bigger yield of sorted cells if automatic instrument is used for a comparable time as FACS that usually resulted with a lower concentrated cell fraction. Moreover, the MACS technique for the removal of PI positive dead cells has been already patented (Fox et al., 2012), although their practical outcomes are unknown. Anyway, the alternative to the FACS or MACS sorting of $\mathrm{PI}^{+}$cells could be another commercially available product named Dead Cell Removal Kit (Miltenyi Biotec, Germany) for removal of dead cells via MACS. However, the potential use of this kit for the improvement of the farm animal semen quality has to be explored yet. In any case, it is obvious that several factors such as the yield, purity, toxic effect or even species etc. should be taken into consideration before one's decided for the sorting strategy.

\section{CONCLUSION}

In conclusion, the improvement of sperm quality by FACS sorting of rabbit spermatozoa using FACSMelody instrument seems to be possible. However, several aspects of the sorting have to be taken into consideration. At first, sterile PBS should be used as a sheath fluid and collection tubes should be washed with FBS prior the sorting. An interesting fact is that the dyes are somehow washed out from the stained cells during the sorting probably due to the higher pressure necessary for sorting. On the other hand, the sorting pressure at $23 \mathrm{psi}$ did not significantly affect the viability of negatively sorted spermatozoa. However, the ram sperm sorting is still a big challenge in order to improve the semen quality of individual breeding male and thus for example increase the cryopreservation efficiency of the insemination doses due to the high physiological sensitivity of ram spermatozoa. For further optimisation of sorting experiments we therefore suggest to try on the use of some specific ram sperm diluent for staining procedure as well as a liquid for sorted sperm in the collection tubes to minimise any additional negative effect on the sperm viability and motility. Moreover, a fertilizing ability of the sorted sperm of any animal species should be assessed after each kind of sorting.

Acknowledgments: The study was financially supported by the Slovak Research and Development Agency (No. APVV-17-0124), by the Scientific Grant Agency (grant no. VEGA 1/0049/19) and Educational Grant Agency of the Ministry of Education, Science, Research and Sport of the Slovak Republic and the Slovak Academy of Sciences (grant no. KEGA 026SPU-4/2018). The authors wish to especially thank Ms. P. Ulická from the distribution company Bio G, spol. s r.o. (Slovak Republic) and Dr. J. Šinkora from BD Biosciences (Czech Republic) for their technical assistance and for lending the FACSMelody instrument.

\section{REFERENCES}

Aitken, R. J., \& Koppers, A. J. (2011). Apoptosis and DNA damage in human spermatozoa. Asian journal of andrology, 13(1), 36. https://doi.org/10.1038/aja.2010.68

Auger, J., Leonce, S., Jouannet, P., \& Ronot, X. (1993). Flow cytometric sorting of living, highly motile human spermatozoa based on evaluation of their mitochondrial activity. Journal of Histochemistry \& Cytochemistry, 41(8), 12471251. https://doi.org/10.1177/41.8.8331289

Aziz, N., Said, T., Paasch, U., \& Agarwal, A. (2007). The relationship between human sperm apoptosis, morphology and the sperm deformity index. Human Reproduction, 22(5), 1413-1419. https://doi.org/10.1093/humrep/dem016

Baláži, A., Vašíček, J., Svoradová, A., Macháč, M., Jurčík, R., Huba, J., Pavlík, I., \& Chrenek, P. (2020). Comparison of three different methods for the analysis of ram sperm concentration. Slovak Journal of Animal Science, 53(2), 53-58.

Campos-Chillon, L. F., \& De La Torre, J. F. (2003). Effect of concentration of sexed bovine sperm sorted at 40 and $50 \mathrm{psi}$ on developmental capacity of in vitro produced embryos. Theriogenology, 59, 506. https://doi.org/10.1016/S0093691X(02)01253-0

da Silva, C. B., Ferrusola, C. O., Rodriguez, A. M., Bolanos, J. G., Davila, M. P., Morrell, J. M., ... \& Pena, F. J. (2013). Sex sorting increases the permeability of 
the membrane of stallion spermatozoa. Animal reproduction science, 138(3-4), 241-251. https://doi.org/10.1016/j.anireprosci.2013.02.021

De Geyter, C., Gobrecht-Keller, U., Ahler, A., \& Fischer, M. (2019). Removal of DNA-fragmented spermatozoa using flow cytometry and sorting does not improve the outcome of intracytoplasmic sperm injection. Journal of Assisted Reproduction and Genetics, 36(10), 2079-2086. https://doi.org/10.1007/s10815019-01571-1

De Graaf, S. P., Evans, G., Maxwell, W. M. C., \& O’Brien, J. K. (2006). In vitro characteristics of fresh and frozen-thawed ram spermatozoa after sex sorting and re-freezing. Reproduction, Fertility and Development, 18(8), 867-874. https://doi.org/10.1071/RD06061

De Graaf, S. P., Evans, G., Maxwell, W. M. C., Downing, J. A., \& O'Brien, J. K. (2007). Successful low dose insemination of flow cytometrically sorted ram spermatozoa in sheep. Reproduction in Domestic Animals, 42(6), 648-653. https://doi.org/10.1111/j.1439-0531.2006.00837.x

Enciso, M., Pieczenik, G., Cohen, J., \& Wells, D. (2012). Development of a novel synthetic oligopeptide for the detection of DNA damage in human spermatozoa. Human reproduction, 27(8), 2254-2266. https://doi.org/10.1093/humrep/des201

Fox, D. N., Sadeh, T., Ottenberg, M. J., Melnik, K. M., \& Schenk, J. L. (2012) U.S. Patent Application No. 13/398,467.

Frijters, A. C. J., Mullaart, E., Roelofs, R. M. G., Van Hoorne, R. P., Moreno, J. F., Moreno, O., \& Merton, J. S. (2009). What affects fertility of sexed bull semen more, low sperm dosage or the sorting process?. Theriogenology, 71(1), 64-67. https://doi.org/10.1016/j.theriogenology.2008.09.025

Garcia, E. M., Vazquez, J. M., Parrilla, I., Calvete, J. J., Sanz, L., Caballero, I., \& Martinez, E. A. (2007). Improving the fertilizing ability of sex sorted boar $\begin{array}{lll}\text { spermatozoa. } & \text { Theriogenology, } & \text { 771-778 }\end{array}$ https://doi.org/10.1016/j.theriogenology.2007.06.006

Garner, D. L. (2006). Flow cytometric sexing of mammalian sperm. Theriogenology, $65(5)$

https://doi.org/10.1016/j.theriogenology.2005.09.009

Gawlitta, D., Oomens, C. W., Baaijens, F. P., \& Bouten, C. V. (2004). Evaluation of a continuous quantification method of apoptosis and necrosis in tissue cultures Cytotechnology, 46(2-3), 139-150. https://doi.org/10.1007/s10616-005-2551-7

Gosálvez, J., Núñez, R., Fernández, J. L., López-Fernández, C., \& Caballero, P. (2011). Dynamics of sperm DNA damage in fresh versus frozen-thawed and gradient processed ejaculates in human donors. Andrologia, 43(6), 373-377. https://doi.org/10.1111/j.1439-0272.2010.01022.x

Grunewald, S., Paasch, U., \& Glander, H. J. (2001). Enrichment of non-apoptotic human spermatozoa after cryopreservation by immunomagnetic cell sorting. Cell and tissue banking, 2(3), 127-133. https://doi.org/10.1023/A:1020188913551

Hamilton, T. R. D. S., Mendes, C. M., Castro, L. S. D., Assis, P. M. D., Siqueira, A. F. P., Delgado, J. D. C., ... \& Visintin, J. A. (2016). Evaluation of lasting effects of heat stress on sperm profile and oxidative status of ram semen and epididymal sperm. Oxidative Medicine and Cellular Longevity, 2016. https://doi.org/10.1155/2016/1687657

Idziorek, T., Estaquier, J., De Bels, F., \& Ameisen, J. C. (1995). YOPRO-1 permits cytofluorometric analysis of programmed cell death (apoptosis) without interfering with cell viability. Journal of immunological methods, 185(2), 249258. https://doi.org/10.1016/0022-1759(95)00172-7

Johnson, L. A. (2000). Sexing mammalian sperm for production of offspring: the state-of-the-art. Animal reproduction science, 60, 93-107. https://doi.org/10.1016/S0378-4320(00)00088-9

Johnson, L. A., Cran, D. G., \& Polge, C. (1994). Recent advances in sex preselection of cattle: Flow cytometric sorting of X-\& Y-chromosome bearing sperm based on DNA to produce progeny. Theriogenology, 41(1), 51-56. https://doi.org/10.1016/S0093-691X(05)80048-2

Johnson, L. A., Flook, J. P., \& Hawk, H. W. (1989). Sex preselection in rabbits: live births from X and Y sperm separated by DNA and cell sorting. Biology of Reproduction, 41(2), 199-203. https://doi.org/10.1095/biolreprod41.2.199

Johnson, L. A., Welch, G. R., \& Rens, W. (1999). The Beltsville sperm sexing technology: high-speed sperm sorting gives improved sperm output for in vitro fertilization and AI. Journal of Animal Science, 77(suppl_2), 213-220. https://doi.org/10.2527/1999.77suppl_2213x

Kuželová, L., Vašíček, J., Rafay, J., \& Chrenek, P. (2017). Detection of macrophages in rabbit semen and their relationship with semen quality. Theriogenology,

97,

$148-153$

\section{https://doi.org/10.1016/j.theriogenology.2017.04.032}

Leahy, T., Marti, J. I., Crossett, B., Evan, G., \& Maxwell, W. M. C. (2011). Twodimensional polyacrylamide gel electrophoresis of membrane proteins from flow cytometrically sorted ram sperm. Theriogenology, 75(5), 962-971. https://doi.org/10.1016/j.theriogenology.2010.11.003

Leahy, T., Marti, J. I., Mendoza, N., Pérez-Pé, R., Muiño-Blanco, T., CebriánPérez, J. A., ... \& Maxwell, W. M. C. (2010). High pre-freezing dilution improves post-thaw function of ram spermatozoa. Animal reproduction science, 119(1-2), 137-146. https://doi.org/10.1016/j.anireprosci.2009.12.003

Martin, G., Cagnon, N., Sabido, O., Sion, B., Grizard, G., Durand, P., \& Levy, R.

(2007). Kinetics of occurrence of some features of apoptosis during the cryopreservation process of bovine spermatozoa. Human reproduction, 22(2), 380-388. https://doi.org/10.1093/humrep/del399

Martin, G., Sabido, O., Durand, P., \& Levy, R. (2004). Cryopreservation induces an apoptosis-like mechanism in bull sperm. Biology of Reproduction, 71(1), 2837. https://doi.org/10.1095/biolreprod.103.024281

Mendoza, N., Casao, A., Pérez-Pé, R., Cebrián-Pérez, J. A., \& Muiño-Blanco, T. (2013). New insights into the mechanisms of ram sperm protection by seminal plasma proteins. Biology of reproduction, 88(6), 149-1. https://doi.org/10.1095/biolreprod.112.105650

Morris, I. D., Ilott, S., Dixon, L., \& Brison, D. R. (2002). The spectrum of DNA damage in human sperm assessed by single cell gel electrophoresis (Comet assay) and its relationship to fertilization and embryo development. Human reproduction, 17(4), 990-998. https://doi.org/10.1093/humrep/17.4.990

Mrkun, J., Dolenšek, T., Knific, T., Pišlar, A., Kosec, M., Kos, J., \& Zrimšek, P. (2014). Elimination of apoptotic boar spermatozoa using magnetic activated cell sorting. Acta Veterinaria Brno, 83(1), 13-18. https://doi.org/10.2754/avb201483010013

Oseguera-López, I., Ruiz-Díaz, S., Ramos-Ibeas, P., \& Pérez-Cerezales, S. (2019). Novel techniques of sperm selection for improving IVF and ICSI outcomes. Frontiers in Cell and Developmental Biology, 7. https://doi.org/10.3389/fcell.2019.00298

Quan, G. B., Ma, Y., Li, J., Wu, G. Q., Li, D. J., Ni, Y. N., ... \& Hong, Q. H. (2015). Effects of Hoechst33342 staining on the viability and flow cytometric sex-sorting of frozen-thawed ram sperm. Cryobiology, 70(1), 23-31. https://doi.org/10.1016/j.cryobiol.2014.11.002

Ribeiro, S. C., Sartorius, G., Pletscher, F., de Geyter, M., Zhang, H., \& de Geyter, C. (2013). Isolation of spermatozoa with low levels of fragmented DNA with the use of flow cytometry and sorting. Fertility and sterility, 100(3), 686694. https://doi.org/10.1016/j.fertnstert.2013.05.030

Robinson, L., Gallos, I. D., Conner, S. J., Rajkhowa, M., Miller, D., Lewis, S., \& Coomarasamy, A. (2012). The effect of sperm DNA fragmentation on miscarriage rates: a systematic review and meta-analysis. Human reproduction, 27(10), 2908-2917. https://doi.org/10.1093/humrep/des261

Said, T., Agarwal, A., Grunewald, S., Rasch, M., Baumann, T., Kriegel, C., ... \& Paasch, U. (2006). Selection of nonapoptotic spermatozoa as a new tool for enhancing assisted reproduction outcomes: an in vitro model. Biology of reproduction, 74(3), 530-537. https://doi.org/10.1095/biolreprod.105.046607

Schulte, R. T., Ohl, D. A., Sigman, M., \& Smith, G. D. (2010). Sperm DNA damage in male infertility: etiologies, assays, and outcomes. Journal of assisted reproduction and genetics, 27(1), 3-12. https://doi.org/10.1007/s10815-009. 9359-x

Seidel Jr, G. E. (2007). Overview of sexing sperm. Theriogenology, 68(3), $443-$ 446. https://doi.org/10.1016/j.theriogenology.2007.04.005

Seidel Jr, G. E., Brink, Z., \& Schenk, J. L. (2003). Use of heterospermic insemination with fetal sex as the genetic marker to study fertility of sexed sperm. Theriogenology, 59, 515. https://doi.org/10.1016/S0093-691X(02)01253$\underline{\mathbf{0}}$

Sharpe, J. C., \& Evans, K. M. (2009). Advances in flow cytometry for sperm sexing. Theriogenology, 71(1), 4-10. https://doi.org/10.1016/j.theriogenology.2008.09.021

Suh, T. K., Schenk, J. L., \& Seidel Jr, G. E. (2005). High pressure flow cytometric sorting damages sperm. Theriogenology, 64(5), 1035-1048 https://doi.org/10.1016/j.theriogenology.2005.02.002

Varshini, J., Srinag, B. S., Kalthur, G., Krishnamurthy, H., Kumar, P., Rao, S. S., $\&$ Adiga, S. K. (2012). Poor sperm quality and advancing age are associated with increased sperm DNA damage in infertile men. Andrologia, 44, 642-649. https://doi.org/10.1111/j.1439-0272.2011.01243.x

Vasicek, J., Pivko, J., \& Chrenek, P. (2014). Reproductive performance of New Zealand White rabbits after depletion of apoptotic spermatozoa. Folia biologica, 62(2), 109-117. https://doi.org/10.3409/fb62 2.109

Vazquez, J. M., Parrilla, I., Roca, J., Gil, M. A., Cuello, C., Vazquez, J. L., \& Martinez, E. A. (2009). Sex-sorting sperm by flow cytometry in pigs: issues and perspectives. Theriogenology, 71(1), https://doi.org/10.1016/j.theriogenology.2008.09.044

Virro, M. R., Larson-Cook, K. L., \& Evenson, D. P. (2004). Sperm chromatin structure assay $(\mathrm{SCSA} \circledast)$ parameters are related to fertilization, blastocys development, and ongoing pregnancy in in vitro fertilization and intracytoplasmic sperm injection cycles. Fertility and sterility, 81(5), 1289-1295. https://doi.org/10.1016/j.fertnstert.2003.09.063

Zhao, H., Oczos, J., Janowski, P., Trembecka, D., Dobrucki, J., Darzynkiewicz, Z., \& Wlodkowic, D. (2010). Rationale for the real-time and dynamic cell death assays using propidium iodide. Cytometry. Part A : the journal of the International Society for Analytical Cytology, 77(4), 399-405. https://doi.org/10.1002/cyto.a.20867

Zini, A., Boman, J. M., Belzile, E., \& Ciampi, A. (2008). Sperm DNA damage is associated with an increased risk of pregnancy loss after IVF and ICSI: systematic review and meta-analysis. Human reproduction, 23(12), 2663-2668. https://doi.org/10.1093/humrep/den321 
Zorn, B., Golob, B., Ihan, A., Kopitar, A., \& Kolbezen, M. (2012). Apoptotic sperm biomarkers and their correlation with conventional sperm parameters and male fertility potential. Journal of assisted reproduction and genetics, 29(4), 357364. https://doi.org/10.1007/s10815-012-9718-x 